\title{
Absceso cerebral complicado con ruptura intraventricular: reporte de dos casos y revisión de la literatura
}

\author{
Intraventricular rupture of brain abscess: report of two cases and review \\ of the literature
}

\author{
Jorge Alonso Ramírez Quiñones ${ }^{1}$, María Elena Novoa Mosquera ${ }^{2}$ \\ ${ }^{1}$ Médico residente de Neurología, Instituto Nacional de Ciencias Neurológicas. \\ ${ }^{2}$ Médico neurólogo, Departamento de Enfermedades Neurovasculares y Metabólicas, Instituto Nacional de Ciencias Neurológicas.
}

\begin{abstract}
Resumen
El absceso cerebral es una patología infecciosa infrecuente cuyo diagnóstico oportuno y tratamiento adecuado conducen a un buen pronóstico en la mayoría de los casos. La ruptura intraventricular es una complicación rara del absceso cerebral con elevada mortalidad; se caracteriza por compromiso brusco de la conciencia, con aparición de signos de irritación meníngea y hallazgos radiológicos compatibles con un drenaje de secreción purulenta hacia los ventrículos. El tratamiento incluye la administración de antibióticos intravenosos e intratecales asociados a una rápida intervención quirúrgica para drenaje del absceso y del contenido intraventricular. Presentamos dos casos de absceso cerebral complicado con ruptura intraventricular, que mostraron caracteristicas clínicas y radiológicas propias de esta condición, con evolución favorable solo con tratamiento antibiótico endovenoso durante seis a diez semanas, respectivamente.
\end{abstract}

Palabras clave: Absceso cerebral; meningitis; ventriculitis cerebral (fuente: DeCS BIREME).

Abstract

Brain abscess is an infrequent infectious disease that has a good prognosis in most of the cases with timely diagnosis and suitable treatment. Intraventricular rupture is a rare complication of the brain abscess with high mortality. It is characterized by a sudden consciousness compromise with signs of meningeal irritation and radiological findings compatible with purulent drainage into the ventricles. Treatment includes administration of intravenous and intrathecal antibiotics associated to prompt surgical intervention to drain the abscess and intraventricular content. Two cases of brain abscess complicated with intraventricular rupture showing clinical and radiological features typical of this condition and favorable outcome with intravenous antibiotic treatment during six and ten weeks respectively are reported.

Keywords: Brain abscess; meningitis; cerebral ventriculitis (source: MeSH NLM).

An Fac med. 2014;75(2):159-63 / doi: http://dx.doi.org/10.15381/anales.v75i2.8388

\section{INTRODUCCIÓN}

El absceso cerebral (AC) es una patología infecciosa poco frecuente que afecta el parénquima cerebral. En los últimos años, los avances tanto en el diagnóstico como en el tratamiento han producido una disminución significativa en la tasa de mortalidad de esta patología ${ }^{(1)}$. La ruptura intraventricular del absceso cerebral es una complicación grave, con alta tasa de mortalidad y un elevado porcentaje de secuelas neurológicas entre los sobrevivientes ${ }^{(2)}$. Esta ruptura tiene características clínicas y radiológicas particulares y el tratamiento debe combinar procedimientos tanto médicos como quirúrgicos para obtener mejores resultados. Comunicamos dos casos de AC complicados con ruptura intraventricular en pacientes inmunocompetentes, con estudios de tomografía computarizada (TC) y resonancia magnética (RM) cerebral, que respondieron de forma satisfactoria al tratamiento antibiótico endovenoso sin presentar secuelas neurológicas al alta.

\section{REPORTE DE CASOS}

\section{CASO 1}

Paciente de sexo masculino de 16 años de edad, procedente de Ancash, con antecedente de sinusitis maxilar, 


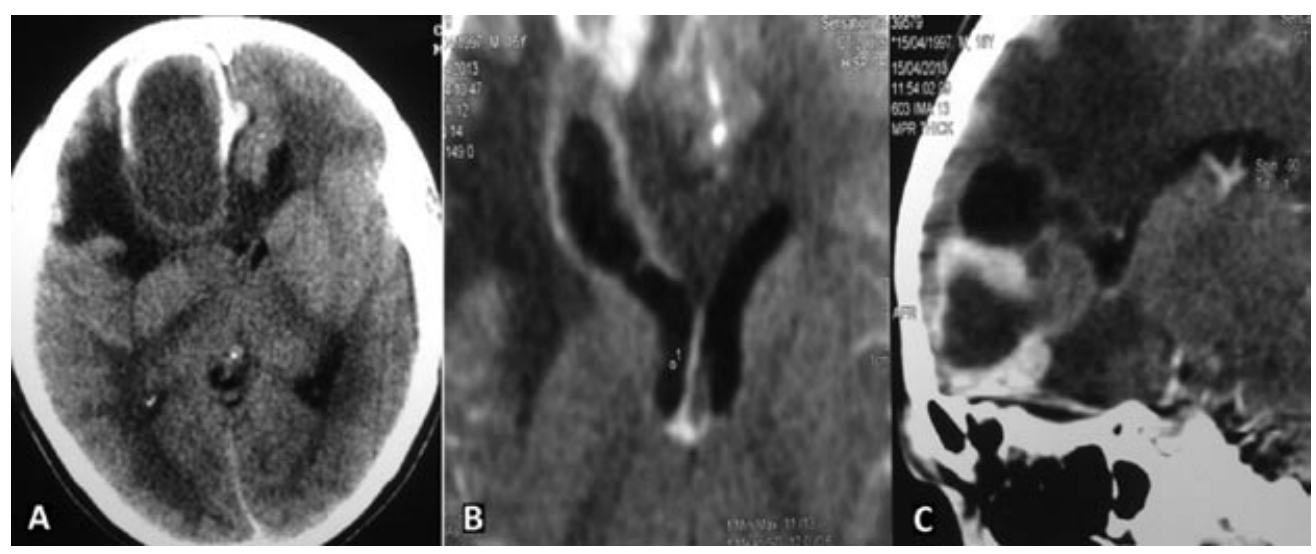

Figura 1. (A) TC cerebral sin contraste, en corte axial, que muestra masa en región frontal derecha con realce del contorno en anillo y gran edema alrededor. Se aprecia que el grosor del contorno es menor en el lado ventricular que en el cortical. (B) TC con contraste en corte coronal que evidencia comunicación de dicha lesión con el ventrículo lateral, con realce de pared ventricular. (C) TC con contraste en corte sagital que muestra comunicación de lesión con ventrículo lateral.

acudió con tiempo de enfermedad de dos semanas, inicio insidioso y curso progresivo, caracterizado por cefalea holocraneana tipo opresiva de moderada intensidad asociada a náuseas y vómitos, irritabilidad, sin fiebre. Un día antes del ingreso presentó crisis tónicoclónica generalizada. En la evaluación al ingreso, el paciente estaba despierto, orientado y sin focalización neurológica. La analítica sanguínea no mostró alteraciones. La TC cerebral reveló imagen hipodensa frontal derecha, con gran efecto de masa (figura 1A), captación de contraste en anillo rodeado de un área extensa de edema, hallazgos sugerentes de absceso cerebral o neoplasia intracraneal. A los tres días, el paciente se tornó estuporoso, con signos de irritación meníngea. La TC cerebral de control mostró disminución del volumen de la masa con aparente comunicación con el asta frontal del ventrículo lateral derecho (figuras $1 \mathrm{~B}$, 1C y 2A). La RM evidenció, en los protocolos T2, FLAIR (fluid attenuated inversion recovery) (figura $2 \mathrm{~B}$ ) y tras la aplicación de contraste, presencia de contenido hiperintenso en cavidad ventricular, con realce de sus paredes.
Se diagnosticó un AC complicado con ruptura intraventricular; se trasladó al paciente a la unidad de cuidados intensivos y se inició tratamiento antibiótico endovenoso de amplio espectro. No se realizó intervención neuroquirúrgica. Luego de una semana de tratamiento mejoró su nivel de conciencia y luego de cuatro semanas la TC de control evidenció marcada disminución de volumen del AC sin compromiso ventricular (figura 2C). El tratamiento antibiótico continuó durante diez semanas. El paciente fue dado de alta sin secuelas neurológicas.
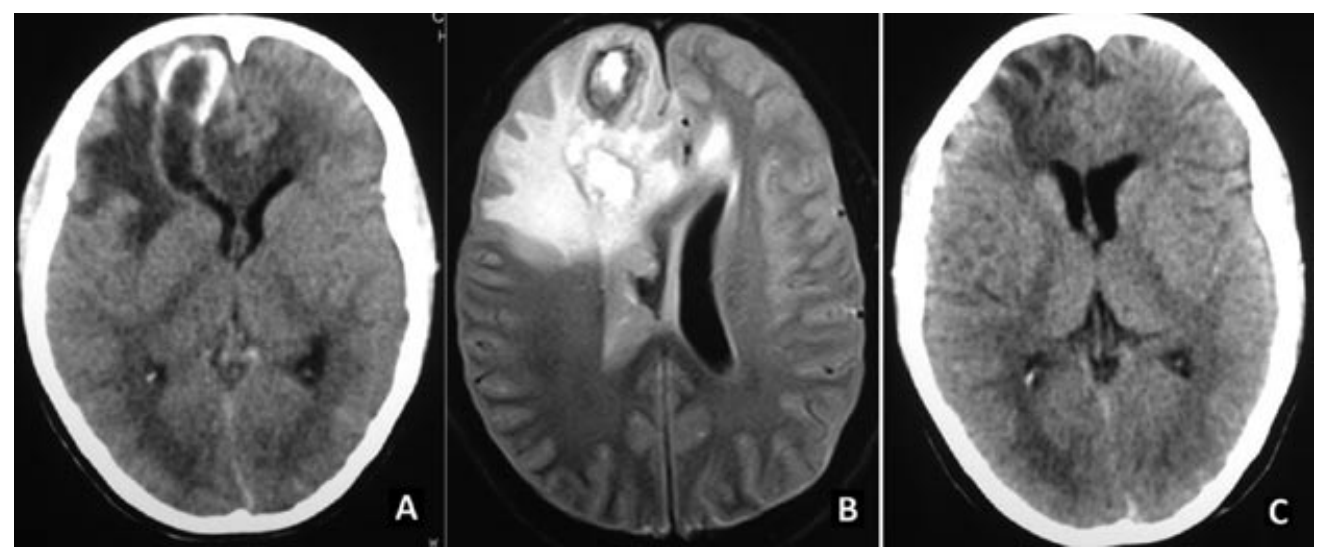

Figura 2. (A) TC sin contraste en corte axial, que evidencia comunicación de lesión con ventrículo lateral e incremento de densidad del LCR en el ventrículo lateral derecho. (B) Imagen de resonancia magnética en protocolo FLAIR corte axial, que muestra material hiperintenso en ventrículo lateral derecho. (C) TC sin contraste en corte axial de control que evidencia disminución de volumen de lesión y ausencia de compromiso intraventricular. 


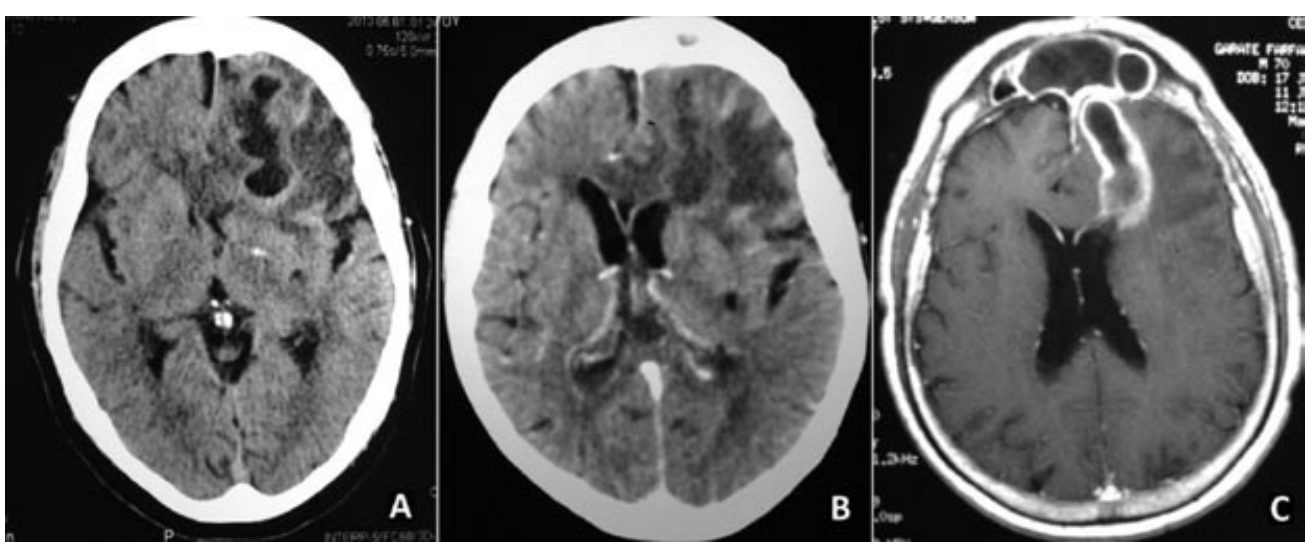

Figura 3. (A) TC sin contraste, corte axial, que evidencia masa trilobulada frontal izquierda con escaso realce del contorno, rodeada de área de edema con efecto de masa. (B) TC con contraste corte axial que muestra aparente comunicación de dicha lesión con ventrículo lateral izquierdo, realce de paredes y depósito de material hiperdenso en ambos ventrículos laterales. (C) Imagen de resonancia magnética en protocolo T1 con gadolinio, corte axial, que evidencia comunicación entre lesión y ventrículo lateral izquierdo, con realce de pared ventricular asociado a lesión en seno frontal, compatible con mucopiocele.

\section{CASO 2}

Paciente de sexo masculino de 70 años de edad, procedente de Lima, con antecedente de traumatismo encefalocraneano en región frontal un año atrás. Acudió con tiempo de enfermedad de un día, inicio brusco, caracterizado por episodio de crisis tónico-clónica generalizada, luego del cual permanece con cefalea holocraneana de intensidad moderada. Al ingreso el paciente estaba despierto, orientado, sin signos de focalización neurológica. No se encontró alteraciones en la analítica sanguínea. La TC cerebral mostró imagen hipodensa lobulada frontal izquierda, con efecto de masa (figura 3A), tenue captación de contraste en anillo y rodeada de un área extensa de edema, compatible con posible neoplasia intracraneal. Al segundo día del ingreso, empeoró el nivel de conciencia y aparecieron signos de irritación meníngea. La TC cerebral de control reveló disminución de volumen y borramiento del borde ventricular de la lesión (figura 3B). La RM evidenció comunicación de la lesión con cavidad ventricular, con captación de contraste en anillo y realce de las paredes ventriculares, asociado a una lesión de seno frontal compatible con mucopiocele (figura 3C). Se diagnosticó AC complicado con ruptura intraventricular y se inició tratamiento antibiótico endovenoso de amplio espectro. No se realizó intervención neuroquirúrgica. Una semana después, el paciente se hallaba despierto, con disminución de los síntomas de irritación meníngea. A las tres semanas, la TC de control mostró disminución de volumen de la lesión, con presencia de contenido hiperdenso con relación al líquido cefalorraquídeo (LCR) en cavidades ventriculares (figura 4). Se continuó tratamiento antibiótico por seis semanas y el paciente fue dado de alta sin secuelas neurológicas.

\section{DISCUSIÓN}

El absceso cerebral es un proceso infeccioso focal del parénquima cerebral que se inicia con un área localizada de cerebritis y progresa a una colección de pus rodeada por una cápsula bien vascularizada ${ }^{(3)}$. La ruptura intraventricular del absceso cerebral es una complicación rara y es el resultado del drenaje del contenido del AC hacia la cavidad ventricular, produciendo ventriculitis y meningitis ${ }^{(4)}$. La incidencia aproximada de $\mathrm{AC}$ es de 4 casos/millón de habitantes/año, con tendencia a disminuir en los últimos años con los avances en el diagnóstico y tratamiento, excepto en pacientes inmunocomprometidos.
Existe una relación hombre-mujer de 2-3:1 y en la mayoría de casos se presenta durante la tercera década de la vida, pudiendo manifestarse a cualquier edad. El AC representa el 8\% de las masas intracraneales en países en vías de desarrollo y de $1 \%$ a $2 \%$ en los desarrollados. La mortalidad oscila entre $5 \%$ a $15 \%$ de los casos, excepto en la ruptura intraventricular del absceso cerebral, situación en que la mortalidad oscila entre $38 \%$ a $84 \%$, con tasas altas de discapacidad en los sobrevivientes ${ }^{(1,2)}$.

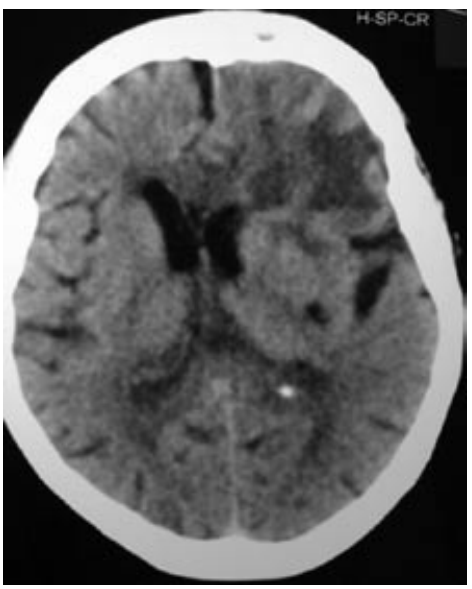

Figura 4. TC sin contraste, corte axial, que evidencia disminución de volumen de la lesión y del área de edema, con compromiso de los ventrículos laterales. 
Diversos organismos -entre bacterias, hongos y parásitos- producen el AC. La etiología depende del sitio primario de infección, la edad y el estado inmunológico de la persona, siendo las bacterias los agentes más frecuentes en inmunocompetentes y agentes oportunistas en inmunocomprometidos. Un agente infeccioso accede al parénquima cerebral mediante tres mecanismos: diseminación por contigüidad, diseminación hematógena o por diseminación directa luego de una cirugía o trauma ${ }^{(3,5)}$. La diseminación por contigüidad representa entre 20 y $70 \%$ de los casos ${ }^{(6)}$. Las infecciones más frecuentes son la otitis media crónica, mastoiditis, sinusitis frontal o etmoidal y las infecciones dentarias, alcanzando el parénquima cerebral por compromiso óseo o mediante las venas emisarias ${ }^{(7)}$. Las otomastoiditis causan AC en el lóbulo temporal y el cerebelo, mientras que las infecciones dentarias y las sinusitis frontales y etmoidales lo hacen en el lóbulo frontal predominantemente ${ }^{(1,3,7)}$. Este mecanismo se aplica en el caso 2, por el hallazgo de una lesión compatible con mucopiocele en seno frontal y por la localización del AC. La diseminación hematógena representa el 20\% de los casos y se produce con mayor frecuencia en pacientes con endocarditis infecciosa, infecciones cutáneas, pulmonares, abdominales o pélvicas. Las cardiopatías congénitas cianóticas o las malformaciones arteriovenosas pulmonares predisponen a este tipo de diseminación ${ }^{(6)}$. Los AC producidos por diseminación hematógena pueden ser únicos o múltiples; se ubican en la unión entre la sustancia gris y blanca, usualmente en el territorio de la arteria cerebral media ${ }^{\left({ }^{8}\right)} \mathrm{y}$ tienen mayor riesgo de ruptura intraventricular del absceso cerebral ${ }^{(1)}$. La contaminación directa representa entre $5 \%$ y $10 \%$ de los casos y con tendencia a incrementarse ${ }^{(9)}$. En el caso 1 no se encontró una fuente de infección manifiesta, situación que según Roche y col. se observa en 20\% de los casos ${ }^{(5)}$.

El agente infeccioso en el parénquima cerebral produce alteración de la permeabilidad vascular local y reclutamiento de células inflamatorias, que originan cambios que son divididos en cuatro etapas histopatológicas ${ }^{(1,3)}$. La primera, denominada cerebritis temprana, abarca desde el día uno al día tres y se caracteriza por una respuesta inflamatoria perivascular que rodea un centro necrótico incipiente. En la segunda etapa o cerebritis tardía (desde el día cuatro al nueve) existe un centro necrótico bien formado, con aparición de fibroblastos y neovascularización en la periferie. La tercera etapa o capsular temprana (del día diez al trece) se caracteriza por una disminución en el tamaño del centro necrótico, con la formación de una capa bien delimitada de fibroblastos y depósito de reticulina, siendo este depósito mayor en el lado cortical que en el lado ventricular del absceso ${ }^{(3)}$, característica que explica la vulnerabilidad de los abscesos a romperse hacia los ventrículos. La última etapa o capsular tardía (a partir del día catorce) se caracteriza por la formación de una cápsula con engrosamiento progresivo y depósito de colágeno ${ }^{(3)}$. La localización más frecuente es el lóbulo frontal ${ }^{(1,5,10)}$, al igual que en nuestros casos, siendo los más susceptibles de ruptura los ubicados en el lóbulo occipital ${ }^{(11)}$.

Las manifestaciones clínicas del AC al inicio pueden ser inespecíficas y dependen de su tamaño, ubicación, de la virulencia del agente infeccioso y de la condición previa del huésped ${ }^{(10)}$. De forma frecuente, se manifiestan como un cuadro de hipertensión endocraneana, un déficit neurológico focal o un cuadro infeccioso sistémico ${ }^{(12)}$. Los síntomas comúnmente encontrados son cefalea (70\% de casos), alteración del nivel de conciencia, náuseas y/o vómitos y fiebre ${ }^{(1,5,10)}$. De nuestros pacientes, ninguno presentó fiebre, demostrando que no es un síntoma patognomónico del AC. Los signos dependen de la región cerebral afectada y/o de una complicación, siendo los más frecuentes déficit motor, crisis epilépticas y meningismo. La ruptura intraventricular del absceso cerebral produce ventriculitis, meningitis y alteraciones en la circulación del líquido cefalorraquídeo y se la sospecha cuando se produce un rápido deterioro del estado general, disminución del estado de conciencia,incrementode la intensidad de la cefalea y aparición de signos de irritación meníngea ${ }^{(2,11)}$. Nuestros casos presentaron súbita alteración del estado de conciencia y aparición de signos meníngeos, probablemente coincidiendo con la ruptura intraventricular.

En el diagnóstico del AC, la analítica sanguínea tiene capacidad limitada. Se puede encontrar leucocitosis, la velocidad de sedimentación globular y la proteína $\mathrm{C}$ reactiva sérica pueden estar elevadas; y los hemocultivos con frecuencia son negativos, tal como ocurrió en estos pacientes. La punción lumbar y el estudio del LCR tienen una utilidad limitada, mostrando cambios inespecíficos y elevando el riesgo de herniación cerebral (3). Los estudios de imágenes son fundamentales en el diagnóstico. La TC en la etapa de cerebritis muestra un área hipodensa que luego de la aplicación de contraste puede no mostrar cambios (cerebritis temprana), adquirir un realce nodular o en anillo (cerebritis tardía), al igual que las etapas capsulares. El AC en etapa capsular es visto en el protocolo T1 de la RM como un área hiperintensa en relación al LCR e hipointensa en relación a la sustancia blanca, rodeada de una cápsula isointensa o hiperintensa, con forma de anillo y con realce tras la administración de contraste. En el protocolo T2 se observa un área isointensa o hiperintensa con relación al LCR, rodeado de una cápsula isointensa o hipointensa con relación a la sustancia blanca. El edema vasogénico que rodea al $\mathrm{AC}$ es hipointenso en $\mathrm{T} 1$ e hiperintenso en $\mathrm{T} 2{ }^{(1,13,14)}$. Todos estos hallazgos fueron observados en nuestros pacientes, pero debido a que existen patologías con hallazgos similares no fue sencillo hacer el diagnóstico de AC. En el protocolo de difusión, el AC muestra una imagen con restricción en la difusión, con realce en anillo asociado a valores disminuidos del coeficiente de difusión aparente (ADC) 
en el mapa $A D C{ }^{(15)}$. En la espectroscopia, el AC muestra niveles elevados de los aminoácidos citosólicos (valina, leucina, isoleucina) y lactato ${ }^{(14)}$. Lee y col. comunicaron que en la RM de pacientes con ruptura intraventricular del absceso cerebral se encuentra realce ependimario (pared ventricular), realce meníngeo, septos y detritus intraventriculares ${ }^{(2)}$, hallazgos presentados también en nuestros casos.

El tratamiento del AC puede ser médico o quirúrgico (incluye la aspiración del contenido y la escisión total del absceso) dependiendo del número, tamaño, localización y etapa evolutiva del absceso, fuente de infección primaria y/o condición previa del paciente ${ }^{(16)}$. Actualmente, el tratamiento incluye la combinación de cirugía (aspiración por estereotaxia) y de tratamiento médico ${ }^{(1,10,16)}$, siendo individualizado en cada escenario. En los casos de ruptura intraventricular del absceso cerebral, el manejo debe incluir una rápida evacuación del absceso, ventriculostomía con lavado ventricular y administración intraventricular e intravenosa de antibióticos ${ }^{(1,2)}$. Se ha encontrado una mortalidad de $67 \%$ en aquellos pacientes que solo reciben tratamiento antibiótico endovenoso sin cirugía (2). Nuestros pacientes no fueron sometidos a intervención neuroquirúrgica debido al profundo compromiso del estado general, pero tuvieron una respuesta favorable con total recuperación y sin secuelas neurológicas solo con antibioticoterapia endovenosa.

En conclusión, presentamos dos casos de ruptura intraventricular del absceso cerebral, complicación infrecuente del AC, que ocurrieron en eda- des extremas de la vida y con respuesta exitosa al tratamiento médico. Se debe sospechar ruptura intraventricular del absceso cerebral en pacientes con diagnóstico de $\mathrm{AC}$ que sufren un deterioro brusco del estado general, compromiso del nivel de conciencia y aparición de signos meníngeos. Se debe confirmar rápidamente el diagnóstico mediante TC y/o RM, ya que, a pesar de la evolución favorable de nuestros casos solo con tratamiento médico, se recomienda un drenaje de urgencia del AC y lavado ventricular asociado a terapia antibiótica intravenosa e intratecal, para disminuir el riesgo de muerte y de secuelas neurológicas.

\section{REFERENCIAS BIBLIOGRÁFICAS}

1. Muzumdar D, Jhawar S, Goel A. Brain abscess: an overview. Int J Surg. 2011;9(2):136-44. doi: http:// dx.doi.org/10.1016/j.ijsu.2010.11.005

2. Lee TH, Chang WN, Su TM, Chang HW, Lui CC, Ho JT, et al. Clinical features and predictive factors of intraventricular rupture in patients who have bacterial brain abscesses. J Neurol Neurosurg Psychiatry. 2007;78(3):303-9. doi: http://dx.doi. org/10.1136/jnnp.2006.097808

3. Kastenbauer S, Pfister $\mathrm{H}$, Wispelwey $\mathrm{B}$, et al Brain Abscess. En: Scheld WM, Whitley RJ, Marra CM. Infections of the central nervous system. Philadelphia: Lippincott Williams \& Wilkins; 2004:479-507.

4. Oshiro S, Ohnishi H, Ohta M, Tsuchimochi H. Intraventricular rupture of Nocardia brain abscess--case report. Neurol Med Chir (Tokyo). 2003;43(7):360-3. doi: http://dx.doi.org/10.2176/nmc.43.360

5. Roche M, Humphreys H, Smyth E, Phillips J, Cunney $R$, McNamara $E$, et al. A twelve-year review of central nervous system bacterial abscesses; presentation and aetiology. Clin Microbiol Infect. 2003;9(8):803-9. doi: http://dx.doi.org/10.1046/ j.1469-0691.2003.00651.x

6. Riddell $\mathrm{J} 4$ th, Shuman EK. Epidemiology of centra nervous system infection. Neuroimaging Clin N Am. 2012;22(4):543-56. doi: http://dx.doi.org/10.1016/j. nic. 2012.05.003

7. Mathisen GE, Johnson JP. Brain abscess. Clin Infect Dis. 1997;25(4):763-79. doi: http://dx.doi. org/10.1086/515541
8. Friedlander RM, Gonzalez RG, Afridi NA, Pfannl R. Case records of the Massachusetts General Hospital. Weekly clinicopathological exercises. Case 16-2003. A 58-year-old woman with left-sided weakness and a right frontal brain mass. N Engl J Med. 2003;348(21):2125-32. doi: http://dx.doi. org/10.1056/NEJMcpc030011

9. Sharma R, Mohandas K, Cooke RPD. Intracranial abscesses: changes in epidemiology and management over five decades in Merseyside. Infection. 2009;37(1):39-43. doi: http://dx.doi.org/10.1007/ s15010-008-7359-X

10. Tseng J-H, Tseng M-Y. Brain abscess in 142 patients: factors influencing outcome and mortality. Surg Neurol. 2006;65(6):557-62. doi: http://dx.doi. org/10.1016/j.surneu.2005.09.029

11. Takeshita M, Kawamata T, Izawa M, Hori T. Prodromal signs and clinical factors influencing outcome in patients with intraventricular rupture of purulent brain abscess. Neurosurgery. 2001;48(2):310-6.

12. Kao PT, Tseng HK, Liu CP, Su SC, Lee CM. Brain abscess: clinical analysis of 53 cases. J Microbiol Immunol Infect. 2003;36(2):129-36.

13. Rath TJ, Hughes M, Arabi M, Shah GV. Imaging of cerebritis, encephalitis, and brain abscess. Neuroimaging Clin N Am. 2012;22(4):585-607. doi: http:// dx.doi.org/10.1016/j.nic.2012.04.002

14. Sarrazin J-L, Bonneville F, Martin-Blondel G. Brain infections. Diagn Interv Imaging. 2012;93(6):473-90. doi: http://dx.doi.org/10.1016/j.diii.2012.04.020

15. Nadal Desbarats L, Herlidou S, de Marco G, Gondry-Jouet C, Le Gars D, Deramond H, et al. Differential MRI diagnosis between brain abscesses and necrotic or cystic brain tumors using the apparent diffusion coefficient and normalized diffusion-weighted images. Magn Reson Imaging. 2003;21(6):645-50. doi: http://dx.doi.org/10.1016/ S0730-725X(03)00084-5

16. Erdoğan E, Cansever T. Pyogenic brain abscess. Neurosurg Focus. 2008;24(6):E2. doi: http://dx.doi. org/10.3171/FOC/2008/24/6/E2

Artículo recibido el 18 de noviembre de 2013 y aceptado para publicación el 6 de enero de 2014.

Fuentes de financiamiento: Autofinanciado.

Declaración de conflictos de interés: Los autores declaran no tener algún conflicto de interés.

Correspondencia:

Dr. Jorge Alonso Ramirez Quiñones

Dirección: Ancash 1271, Lima 1, Perú

Teléfono: 4117700 Anexo 280 - 4582008

Correo electrónico: alonsor45@hotmail.com 